gr-qc/9805010

Class. Quantum Grav. 15 (1998) 3763

\title{
Thiemann transform for gravity with matter fields
}

\author{
Luis J Garay and Guillermo A Mena Marugán \\ Instituto de Matemáticas y Física Fundamental, CSIC, \\ Serrano 121, 28006 Madrid, Spain
}

10 September 1998

\begin{abstract}
The generalized Wick transform discovered by Thiemann provides a well-established relation between the Euclidean and Lorentzian theories of general relativity. We extend this Thiemann transform to the Ashtekar formulation for gravity coupled with spin-1/2 fermions, a non-Abelian Yang-Mills field and a scalar field. It is proved that, on functions of the gravitational and matter phase space variables, the Thiemann transform is equivalent to the composition of an inverse Wick rotation and a constant complex scale transformation of all fields. This result also holds for functions that depend on the shift vector, the lapse function and the Lagrange multipliers of the Yang-Mills and gravitational Gauss constraints, provided that the Wick rotation is implemented by means of an analytic continuation of the lapse. In this way, the Thiemann transform is furnished with a geometric interpretation. Finally, we confirm the expectation that the generator of the Thiemann transform can be determined just from the spin of the fields and give a simple explanation for this fact.
\end{abstract}

PACS numbers: 0460Ds, 0420Fy, 0460Gw

\section{Introduction}

A well-established relation between the Lorentzian and Euclidean theories of vacuum general relativity was found recently by Thiemann [1]. This relation is provided by a generalized Wick transform (which, from now on, we will call the Thiemann transform), defined as an automorphism on the algebra of functions on the gravitational phase space that preserves Poisson brackets. The Thiemann transform generally maps real functions to complex ones, so that it modifies the reality conditions. An extension of this transform to the case when certain types of matter sources (scalar and electromagnetic fields) are present was proposed by Ashtekar [2].

The Thiemann transform was originally formulated in the Ashtekar formalism for general relativity, although it can also be employed, for example, in geometrodynamics and in the triad formalism [2, 3]. In Ashtekar's approach to Lorentzian quantum gravity, it seems necessary to impose complicated reality conditions because the Ashtekar connection is complex for Lorentzian metrics [4, 5]. This problem can be overcome using the Thiemann transform, which maps the Lorentzian to the Euclidean theory of gravity. In fact, the reality conditions are easy to impose in the Euclidean sector since the Ashtekar variables can be chosen to be real in this case ${ }^{\boxplus}$. Furthermore, since the Thiemann trans-

\footnotetext{
${ }^{1}$ This point is discussed in section 5 , including the case when fermions are present.
} 
form maps the Euclidean constraint functionals to the Lorentzian ones, it turns out that this transform allows to obtain the Lorentzian quantum states starting from the Euclidean quantum theory [1, 2]. In this sense, the transform discovered by Thiemann enables one to work with real Ashtekar variables when quantizing general relativity.

The role of the Thiemann transform in quantum gravity is similar to that played by the Wick rotation in quantum field theory in flat spacetime. Both transformations provide a map between a theory that is mathematically difficult to handle and another theory that is, in principle, more manageable. For instance, the Lorentzian path integral in quantum field theory diverges because of the oscillatory character of the integrand $e^{i S}$. The Wick rotation changes this integrand into $e^{-I}$, where $I$ is the Euclidean action. Then, the resulting Euclidean path integral turns out to converge for most matter fields, namely, scalar, Yang-Mills and fermionic fields. In general relativity, the Euclidean path integral is still ill-defined because the gravitational action is unbounded [6, 7]. Nevertheless, it has been proposed that this integral can also be made convergent (at least in the oneloop approximation) by integrating the conformal factor over an appropriate complex contour [7, 8]. Similarly, the problem of dealing with the complicated reality conditions of Lorentzian gravity in the Ashtekar formalism is circumvented with the help of the Thiemann transform. In this way, one arrives at a theory in which Ashtekar variables can be treated as real and that, remarkably, happens to describe Euclidean general relativity.

Despite this analogy between the Thiemann transform and the Wick rotation, it was initially far from clear that a stronger relation between them could actually exist [2]. Indeed, while the Wick rotation can be regarded as an analytic continuation that, from a geometric point of view, is based on a complexification of time, the Thiemann transform, defined as a map on the algebra of functions on phase space, seems to lack a proper geometric interpretation. Such an interpretation would be a helpful guideline for the application of the Thiemann transform to the study of problems of interest in gravity and cosmology.

In order to clarify this point, one of the authors showed recently [3] that the effects of the Thiemann transform on the line element, constraints, and action for vacuum general relativity could be regarded as coming from an inverse Wick rotation and a constant complex scale transformation of the gravitational field. This rescaling amounts to a constant complex conformal transformation of the spacetime metric. As a consequence, it is actually possible to attain a geometric interpretation for the Thiemann transform. Furthermore, it was also proved that the equivalence between the action of the Thiemann transform and the composition of an inverse Wick rotation with a complex conformal transformation applies as well to any function on phase space and to the lapse and shift functions, provided that the Wick rotation is implemented by analytically continuing the lapse from positive real to negative imaginary valuest [10, 11. The main aim of the present paper is to show that this geometric interpretation continues to be valid in the presence of matter sources. In this case, the constant conformal transformation that is involved in the Thiemann transform becomes a constant complex scale transformation of all the fields that is uniquely determined by their respective spins.

Let us briefly review the Hamiltonian formulation for general relativity with matter fields in the Ashtekar formalism. The gravitational phase space can be described by

\footnotetext{
${ }^{2}$ This rotation can be viewed as a continuation in a signature parameter. Some papers that consider this analytic continuation of the signature are are given in [9].
} 
the fields $\left(a_{a}, \sigma^{a}\right)$, defined on a 3-manifold. Here, $a_{a A}{ }^{B}$ is the Ashtekar connection and $\sigma_{A}^{a}{ }^{B}$ are the densitized $S U(2)$ soldering forms [ [if]. As matter content, we will consider a massive scalar field $\phi$, massive spin-1/2 fields $\xi_{A}$ and $\bar{\eta}_{A}$, and a Yang-Mills connection $A_{a}$, where the internal Yang-Mills indices have been suppressed. In addition, we will allow for the presence of a cosmological constant $\Lambda$. The inclusion of this type of matter source was discussed in [5]. A previous analysis of the coupling between fermions and Ashtekar variables can be found in [12].

If we denote the fields $\left\{\phi, \xi, \bar{\eta}, A_{a}\right\}$ as $\left\{q^{k}\right\}$ and their respective canonical momenta $\left\{\pi_{\phi}, \rho, \omega, E^{a}\right\}$ as $\left\{p_{k}\right\}$, the Lorentzian action can be written as 四, 5]:

$$
S=\int d t d^{3} x\left(i \sqrt{2} \sigma^{a} \dot{a}_{a}+\sum_{k} p_{k} \dot{q}^{k}+N \mathcal{S}+\sum_{l} u^{l} \chi_{l}\right),
$$

where traces over Yang-Mills and $S U(2)$ spinor indices have been omitted. We have called $\left\{\chi_{l}\right\}$ to the set of constraints $\left\{\mathcal{G}, G, \mathcal{V}_{a}\right\}$. $\mathcal{G}$ and $G$ denote the Gauss constraints associated with the Ashtekar connection and the Yang-Mills field, respectively, and $\mathcal{V}_{a}$ is the vector constraint (related to spatial diffeomorphisms). The set $\left\{u^{l}\right\}$ denotes the Lagrange multipliers $\left\{\lambda, \beta, N^{a}\right\}$ corresponding to these constraints, $N^{a}$ being the shift vector and $\lambda={ }^{4} a \cdot t, \beta=g\left({ }^{4} A \cdot t\right)$ being the timelike components of the four-dimensional Ashtekar and Yang-Mills connections, the latter rescaled by the Yang-Mills coupling constant $g$ (while the Yang-Mills Gauss constraint has been rescaled by $g^{-1}$ as compared with that in [4]). Finally, $\mathcal{S}$ is the scalar constraint and $N$ is the densitized lapse function (with weight equal to -1 ). These constraints are the generators of the symmetries of the system, which have their origin in geometrodynamics and Yang-Mills theory.

In terms of the phase space variables and Lagrange multipliers, the spacetime line element can be expressed as

$$
d s^{2}=-\sigma^{2} N^{2} d t^{2}+h_{a b}\left(d x^{a}+N^{a} d t\right)\left(d x^{b}+N^{b} d t\right),
$$

where $\sigma=\sqrt{\operatorname{det}\left(\sigma^{a}\right)}$ and the induced three-metric $h_{a b}$ is the inverse of $h^{a b}=-\operatorname{tr}\left(\sigma^{a} \sigma^{b}\right) / \sigma^{2}$.

For the action proposed by Ashtekar, Romano and Tate [4, 5], the explicit form of the constraints can be found in the appendix. This action and constraints lead to the Einstein-Cartan theory, which is quartic in the fermionic variables. Nevertheless, one can attain the Einstein-Dirac formulation, quadratic in fermionic variables, by simply adding

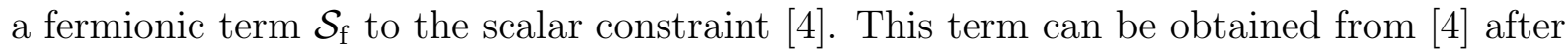
some calculations and has the form

$$
\mathcal{S}_{\mathrm{f}}=-\frac{3}{16}\left(y_{A}{ }^{A} y_{B}{ }^{B}+y_{A B} y^{A B}+y_{A B} y^{B A}\right),
$$

with

$$
y_{A B}=\rho_{A} \xi_{B}+\omega_{A} \bar{\eta}_{B} .
$$

It is worth noting that the gravitational Gauss constraint $\mathcal{G}$ does not depend on any external parameter. On the other hand, the constraints $G$ and $\mathcal{V}_{a}$ depend on the YangMills coupling $g$, and the scalar constraint $\mathcal{S}$ depends on this constant, the fermion mass $m$, the mass squared of the scalar field $\mu^{2}$ and the cosmological constant $\Lambda$. We will refer to these parameters as coupling constants and collectively denote them as $\kappa: \kappa \equiv$ $\left(m, \mu^{2}, g, \Lambda\right)$. 
The rest of the paper is organized as follows. Section 2 is devoted to the Wick rotation in general relativity. Constant scale transformations are studied in section 3 . Section 4 deals with the generalization of the Thiemann transform for gravity with matter fields. We show that the geometric interpretation obtained in [3] for the Thiemann transform in vacuum can be extended to the case when matter sources are present. We conclude and summarize in section 5. In the appendix, we give the detailed expressions of the action and constraints proposed by Ashtekar, Romano and Tate [4, 5].

\section{Wick rotation}

In quantum field theory in flat spacetime, all correlation functions of physical observables can be obtained by means of path integrals of the form $\int \mathcal{D} q e^{i S}$, with $\mathcal{D} q$ being a suitable integration measure for the matter fields. The integrand of this expression is oscillatory and therefore the path integral formally diverges. To avoid this problem, one can perform a Wick rotation, i.e. an analytic continuation $t \rightarrow-i t_{\mathrm{E}}$ from the Lorentzian time $t$ to the Euclidean time $t_{\mathrm{E}}$. Hence, the spacetime metric acquires Euclidean signature. In this way, the Lorentzian configurations are replaced with Euclidean histories and the Lorentzian action $S$ is mapped to $S\left(t \rightarrow-i t_{\mathrm{E}}\right)=i I\left(t_{\mathrm{E}}\right)$, where $I$ is the Euclidean action. It can then be seen that, for integer-spin fields, $I$ is positive definite and therefore the Euclidean path integral $\int \mathcal{D} q e^{-I}$ converges. For fermions, $I$ is complex, but the convergence of the Euclidean path integral is also guaranteed provided that one adopts Berezin integration rules 13 .

In general relativity, one can similarly relate the Lorentzian and Euclidean path integrals by means of a Wick rotation. This gravitational Wick rotation cannot be considered as a rigorously defined transformation that sends each real Lorentzian metric to a real Euclidean one. In fact, the analytic continuation in time of a Lorentzian metric does not generally possess a section in the complexified spacetime on which the metric is real and has Euclidean signature [14]. Nevertheless, it is still possible to regard the Wick rotation as a series of substitution rules that map functions that depend on the real Lorentzian time to functions that depend on the real Euclidean time coordinate. Once this point of view is adopted, the gravitational Wick rotation can be understood as a transformation $R$ that sends, at least formally, the abstract Lorentzian line element to the Euclidean one: $R \circ d s^{2}=d s_{\mathrm{E}}^{2}$, where

$$
d s_{\mathrm{E}}^{2}=\sigma_{\mathrm{E}}^{2} N_{\mathrm{E}}^{2} d t_{\mathrm{E}}^{2}+h_{a b}^{\mathrm{E}}\left(d x^{a}+N_{\mathrm{E}}^{a} d t_{\mathrm{E}}\right)\left(d x^{b}+N_{\mathrm{E}}^{b} d t_{\mathrm{E}}\right) .
$$

In addition, the Lorentzian action $S$ is mapped by this transformation to

$$
R \circ S=i I
$$

There are various ways of implementing this Wick rotation. For instance, we can formally follow the procedure employed in flat spacetime, namely, to perform the analytic continuation of the time parameter $t \rightarrow-i t_{\mathrm{E}}$ [6]. The Lorentzian line element is then transformed into its Euclidean counterpart provided that $R \circ N=N_{\mathrm{E}}, R \circ N^{a}=i N_{\mathrm{E}}^{a}$ and

$$
R \circ \sigma^{a}=\sigma_{\mathrm{E}}^{a} .
$$


Lorentzian and Euclidean quantities depend on $t$ and $t_{\mathrm{E}}$, respectively. On the other hand, the action of $R$ on the matter field variables can be defined by

$$
R \circ q^{k}=q_{\mathrm{E}}^{k}
$$

This definition is a natural generalization of the continuation of the matter fields from the Lorentzian to the Euclidean sector performed in the flat case.

Alternatively, we can rotate the lapse function to the negative imaginary axis [9, 10, 11] and leave the time coordinate and the shift function unchanged, i.e. $R \circ t=t_{\mathrm{E}}$ and

$$
R \circ N=-i N_{\mathrm{E}}, \quad R \circ N^{a}=N_{\mathrm{E}}^{a},
$$

so that the line element is properly transformed under $R$. With this prescription, $\sigma^{a}$ and $q^{k}$ are also left unchanged by the action of $R$.

Note that the transformation laws for $N d t$ and $N^{a} d t$ under the Wick rotation are independent of the specific way in which this rotation is implemented:

$$
R \circ(N d t)=-i N_{\mathrm{E}} d t_{\mathrm{E}}, \quad R \circ\left(N^{a} d t\right)=N_{\mathrm{E}}^{a} d t_{\mathrm{E}} .
$$

Finally, we will understand that the action of $R$ on an integral over time (like, for example, the Lorentzian action) is given by $R \circ \int d t f(t) \equiv \int R \circ[d t f(t)]$, regardless of the prescription adopted for the Wick rotation.

If we define the Euclidean phase space action functional $I$ as

$$
I=\int d t_{\mathrm{E}} d^{3} x\left(\sqrt{2} \sigma_{\mathrm{E}}^{b} \dot{a}_{b}^{\mathrm{E}}+\sum_{k} p_{k}^{\mathrm{E}} \dot{q}_{\mathrm{E}}^{k}+N_{\mathrm{E}} \mathcal{S}_{\mathrm{E}}+\sum_{l} u_{\mathrm{E}}^{l} \chi_{l}^{\mathrm{E}}\right)
$$

we can then deduce the form of the Euclidean matter momenta and the transformation law for the Ashtekar connection by comparing the terms in $R \circ S$ and $i I$ that contain time derivatives:

$$
R \circ p_{k}=i p_{k}^{\mathrm{E}}, \quad R \circ a_{a}=a_{a}^{\mathrm{E}} .
$$

In addition, the Lagrange multipliers $u^{l}$, multiplied by $d t$, transform according to

$$
R \circ\left(u^{l} d t\right)=u_{\mathrm{E}}^{l} d t_{\mathrm{E}}
$$

Indeed, we have already shown that the product $N^{a} d t$ is not modified by the Wick rotation. On the other hand, both $\lambda$ and $\beta$ are defined by the scalar product of the vector $t^{a}$ and a four-dimensional connection $\left({ }^{4} a\right.$ and ${ }^{4} A$, respectively). If we then extend the action of the Wick rotation on the spatial connections $a_{a}$ and $A_{a}$ to their four-dimensional counterparts and take into account that $t^{a} \nabla_{a} t=1$, we see that $\lambda d t=\left({ }^{4} a \cdot t\right) d t$ and $\beta d t=g\left({ }^{4} A \cdot t\right) d t$ are, in fact, unaffected by the Wick rotation, performed either via a continuation of the time coordinate or by rotating the lapse function.

We can also see that the Euclidean constraints must be proportional to the transform of their Lorentzian counterparts under $R$. The factors that relate both sets of constraints can be determined in the following way. If we compare the constraint parts of $R \circ S$ and $i I$ and take into account the transformation laws for $N d t$ and $u^{l} d t$, we obtain

$$
R \circ \mathcal{S}=-\mathcal{S}_{\mathrm{E}}, \quad R \circ \chi_{l}=i \chi_{l}^{\mathrm{E}}
$$


where $\mathcal{S}$ and $\chi_{l}$ depend on the Lorentzian phase space variables, while $\mathcal{S}_{\mathrm{E}}$ and $\chi_{l}^{\mathrm{E}}$ depend on the Euclidean ones. Explicitly, these formulae can be written in the form $\mathcal{S}(R \circ z)=$ $-\mathcal{S}_{\mathrm{E}}\left(z_{\mathrm{E}}\right)$ and $\chi_{l}(R \circ z)=i \chi_{l}^{\mathrm{E}}\left(z_{\mathrm{E}}\right), z$ and $z_{\mathrm{E}}$ denoting the Lorentzian and Euclidean phase space variables, respectively.

Notice that, as far as the action, constraints (possibly including the contribution (11) to the scalar constraint), and canonical variables are concerned, both ways of implementing the Wick rotation give equivalent results. Since, in the Hamiltonian formulation, the system is completely determined by the values of the phase space variables and Lagrange multipliers on any surface of constant time, it is nonetheless most convenient to define the transformation $R$ without making any reference to the time coordinate. This can be achieved by implementing the Wick rotation as a continuation of the lapse function to the negative imaginary axis, thus leaving the time coordinate unaffected.

Let us finally discuss the effect of the Wick rotation on the extrinsic curvature $K_{a b}$. The Ashtekar connection can be written as [4]:

$$
\begin{aligned}
& a_{a}=\Gamma_{a}-i K_{a}+C_{a}, \\
& a_{a}^{\mathrm{E}}=\Gamma_{a}^{\mathrm{E}}+K_{a}^{\mathrm{E}}+i C_{a}^{\mathrm{E}},
\end{aligned}
$$

where (both in the Lorentzian and the Euclidean theories) $K_{a}=\sigma^{b} K_{a b} /(\sqrt{2} \sigma), \Gamma_{a}$ is the spin connection compatible with $\sigma^{a}$ [3], and $C_{a}$ is the fermionic contribution [4]

$$
C_{a}^{A B}=\frac{-i}{4 \sqrt{2}}\left(\sigma_{a}^{A C} y_{C}^{B}+\sigma_{a}^{B C} y_{C}^{A}\right) .
$$

In this formula, $\sigma_{a}$ is the inverse of $\sigma^{a}$ and $y_{A B}$ is defined in equation (2). From the transformation rule for the Ashtekar connection, it is then possible to see that $R \circ K_{a}=$ $i K_{a}^{\mathrm{E}}$. Therefore, the extrinsic curvature transforms under the Wick rotation via

$$
R \circ K_{a b}=i K_{a b}^{\mathrm{E}}
$$

This is precisely the result that one would expect from the expression for the extrinsic curvature that follows from the equations of motion:

$$
K_{a b}=\frac{1}{2 \sigma N}\left[\dot{h}_{a b}-2 D_{(a} N_{b)}\right]
$$

Here, $D_{a}$ is the covariant derivative compatible with $h_{a b}, N_{b}=N^{c} h_{b c}$, and the parentheses denote symmetrization of indices.

\section{Constant scale transformations}

In this section, we will study the behaviour of the gravitational and matter fields under constant scale transformations. With this aim, we first carry out a dimensional analysis of the various fields and coupling constants of the theory.

The line element has dimensions of length squared, i.e. $D\left(d s^{2}\right)=2$. If we describe the spacetime coordinates by means of dimensionless parameters, the metric tensor inherits the dimensions of $d s^{2}$, so that $D\left(\sigma^{a}\right)=2, D(N)=-2$, and $D\left(N^{a}\right)=0$. The spin 
connection $\Gamma_{a}$ compatible with $\sigma^{a}$ has the same dimension as the derivative operator. Since the coordinates are dimensionless, so must be $\Gamma_{a}$. Using equation (1), it is easy to see that the variable $K_{a}=\sigma^{b} K_{a b} /(\sqrt{2} \sigma)$ is also dimensionless. Consequently, we obtain $D\left(a_{a}\right)=0$ in vacuum. Actually, one could have expected this result from the fact that the Ashtekar connection is invariant under constant scale transformations of the metric. It is then not difficult to check that the gravitational action in vacuum has dimensions of area. This conclusion can also be extended to the full action, for gravity with matter sources, taking into account the covariance of the theory and the homogeneity of the action. Thus, we have $D(S)=2$. Note that this fact naturally selects a parameter $l_{*}^{2}$ with units of area for the path integral (whose weight would be $\exp \left(i S / l_{*}^{2}\right)$ ) and that becomes the expansion parameter in the semiclassical approximation. This parameter can be naturally identified with Planck length squared (up to irrelevant numerical factors).

Since $D(S)=2$, the term $p_{k} \dot{q}^{k}$ in the action, which provides the symplectic structure for the matter fields, requires that $D\left(q^{k}\right)+D\left(p_{k}\right)=2$. Noting then that the dimensions of the gravitational variables are not affected by the presence of matter fields and employing the explicit form of the constraints given in the appendix, we can easily calculate the dimensions of all the quantities appearing in the action:

$$
\begin{array}{llll}
D\left(a_{a}\right)=0, & D\left(\sigma^{a}\right)=2, & D\left(q^{k}\right)=(-1)^{2 s_{k}} s_{k}, & D\left(p_{k}\right)=2-(-1)^{2 s_{k}} s_{k}, \\
D\left(u^{l}\right)=0, & D(N)=-2, & D(m)=D(g)=-1, & D\left(\mu^{2}\right)=D(\Lambda)=-2 .
\end{array}
$$

Here, $s_{k}$ is the spin of the matter field $q^{k}$, namely, $s_{\phi}=0, s_{\xi}=s_{\bar{\eta}}=1 / 2$ and $s_{\mathrm{YM}}=1$. Notice that, for the gravitational variables, it is the momentum $\sigma^{a}$ that has the dimension $(-1)^{2 s_{\mathrm{g}}} s_{\mathrm{g}}$ (with $s_{\mathrm{g}}=2$ ) instead of the configuration variable $a_{a}$. This is because the metric (which can be considered as the physically relevant gravitational field) is defined in terms of $\sigma^{a}$ and not in terms of the connection $a_{a}$. Note also that the motivation for modifying the Gauss constraint associated with the Yang-Mills field as well as its Lagrange multiplier by a factor of $g$ becomes now apparent: the dimension of the timelike component of the Yang-Mills connection ${ }^{4} A \cdot t$ is compensated by introducing the factor $g^{-1}$, so that all the Lagrange multipliers, except the densitized lapse function, are dimensionless. On the other hand, the densitized lapse function must have dimension -2 in order to ensure that the line element has the proper dimensions.

For each complex number $\Omega$, let us now consider the constant scale transformations defined by means of the operator $C_{\Omega}$ :

$$
C_{\Omega} \circ f=\Omega^{D(f)} f .
$$

In particular, for the spacetime metric, the action of $C_{\Omega}$ amounts to a constant conformal transformation. It is convenient to split $C_{\Omega}$ into two pieces, $C_{\Omega}^{\mathrm{F}}$ and $C_{\Omega}^{\kappa}$, the former acting only on fields (phase space variables and Lagrange multipliers) and the latter acting on the coupling constants $\kappa=\left(m, \mu^{2}, g, \Lambda\right)$. Since the line element is independent of $\kappa$, we then have $C_{\Omega}^{\mathrm{F}} \circ d s^{2}=\Omega^{2} d s^{2}$. In addition, from the transformation law for the action under $C_{\Omega}, C_{\Omega} \circ S=\Omega^{2} S$, we obtain

$$
C_{\Omega}^{\mathrm{F}} \circ S(\kappa)=\Omega^{2}\left(C_{\Omega}^{\kappa}\right)^{-1} \circ S(\kappa)=\Omega^{2} S[\kappa(\Omega)],
$$

where $\kappa(\Omega) \equiv\left(m \Omega, \mu^{2} \Omega^{2}, g \Omega, \Lambda \Omega^{2}\right)$. Taking then into account the dimensions of the Lagrange multipliers, we conclude that, acting on the constraints, $C_{\Omega}^{\mathrm{F}}$ is

$$
C_{\Omega}^{\mathrm{F}} \circ \chi_{l}(g)=\Omega^{2} \chi_{l}(g \Omega), \quad C_{\Omega}^{\mathrm{F}} \circ \mathcal{S}(\kappa)=\Omega^{4} \mathcal{S}[\kappa(\Omega)] .
$$


Therefore, we see that, in general, $C_{\Omega}^{\mathrm{F}}$ transforms constraints with physical real couplings $\kappa$ into constraints with complex couplings $\kappa(\Omega)$.

\section{Thiemann transform}

The Thiemann transform is an automorphism on the algebra of functions on phase space that preserves the symplectic structure. As such, it can be implemented as a transformation generated via Poisson brackets, as discussed in [1, 2, 3, 15]. None of the transformations considered so far, namely, the Wick rotation $R$ and the constant rescaling of the fields $C_{\Omega}^{\mathrm{F}}$, preserves the symplectic structure. Furthermore, they not only affect the phase space variables but also the Lagrange multipliers. However, if we set $\Omega=e^{i \pi / 4}$ and denote the corresponding operator $C_{\Omega}^{\mathrm{F}}$ by $C^{\mathrm{F}}$, it turns out that the composition $T \equiv C^{\mathrm{F}} \circ R^{-1}$ commutes with the Poisson bracket operation. Indeed, the explicit transformation rules for the Euclidean phase space variables under $T$ are

$$
\begin{array}{ll}
T \circ a_{a}^{\mathrm{E}}=a_{a}, & T \circ q_{\mathrm{E}}^{k}=q^{k} e^{i \pi(-1)^{2 s_{k} s_{k} / 4},} \\
T \circ \sigma_{\mathrm{E}}^{a}=i \sigma^{a}, & T \circ p_{k}^{\mathrm{E}}=p_{k} e^{-i \pi(-1)^{2 s_{k}} s_{k} / 4} .
\end{array}
$$

Using then that the replacement of the Euclidean Ashtekar connection $a_{a}^{\mathrm{E}}$ with the variable $x_{a}^{\mathrm{E}}=a_{a}^{\mathrm{E}}-\Gamma_{a}^{\mathrm{E}}$ (and the change from $i a_{a}$ to $x_{a}=i\left(a_{a}-\Gamma_{a}\right)$ in the Lorentzian theory) amounts to a canonical transformation [4] and recalling that $\Gamma_{a}^{\mathrm{E}}$ is invariant under constant rescalings of $\sigma_{\mathrm{E}}^{a}$, it is not difficult to see that, on phase space, the transformation $T$ is generated via Poisson brackets by

$$
\mathcal{T}=\frac{\pi}{4} \sum_{\alpha}(-1)^{2 s_{\alpha}} s_{\alpha} \int d^{3} x p_{\alpha}^{\mathrm{E}} q_{\mathrm{E}}^{\alpha}
$$

where $\left\{q_{\mathrm{E}}^{\alpha}\right\}$ denotes the set of variables $\left\{-\sqrt{2} \sigma_{\mathrm{E}}^{a}, q_{\mathrm{E}}^{k}\right\}$, their canonically conjugate variables $\left\{x_{a}^{\mathrm{E}}, p_{k}^{\mathrm{E}}\right\}$ are denoted as $\left\{p_{\alpha}^{\mathrm{E}}\right\}$, and $s_{\alpha}$ is the spin of $q_{\mathrm{E}}^{\alpha}$. In the above expression for $\mathcal{T}$, traces over $S U(2)$ and Yang-Mills indices are implicitly assumed. On functions of the Euclidean phase space variables, the action of $T$ is then given by

$$
T \circ f\left(q_{\mathrm{E}}^{\alpha}, p_{\alpha}^{\mathrm{E}}\right)=\mathcal{I} \circ \sum_{n} \frac{i^{n}}{n !}\left\{f\left(q_{\mathrm{E}}^{\alpha}, p_{\alpha}^{\mathrm{E}}\right), \mathcal{T}\right\}_{(n)} .
$$

Here, $\{\cdot, \mathcal{T}\}_{(n)}$ is the $n$th application of the Poisson bracket with $\mathcal{T}$, and $\mathcal{I}$ is an isomorphism between (the algebras of functions on) the Euclidean and Lorentzian phase spaces which, in practical terms, just removes the index E from the canonical variables $\left(q_{\mathrm{E}}^{\alpha}, p_{\alpha}^{\mathrm{E}}\right)$, i.e. $\mathcal{I} \circ\left(q_{\mathrm{E}}^{\alpha}, p_{\alpha}^{\mathrm{E}}\right)=\left(q^{\alpha}, p_{\alpha}\right)$.

In addition, if one adheres to the prescription of performing the Wick rotation by means of a continuation of the lapse function, it is straightforward to check that $T=$ $C^{\mathrm{F}} \circ R^{-1}$ transforms the Euclidean Lagrange multipliers into the Lorentzian ones

$$
T \circ N^{\mathrm{E}}=N, \quad T \circ u_{\mathrm{E}}^{l}=u^{l} .
$$

Note that the definition of $T$ provided by equation (17) can then be extended to the Lagrange multipliers, since they can be considered as functions that are independent of 
the phase space variables and, consequently, have vanishing Poisson brackets with any other function on phase space like, for example, the generator $\mathcal{T}$.

On the other hand, the constraints transform under $T$ according to

$$
T \circ \mathcal{S}_{\mathrm{E}}(\kappa)=\mathcal{S}\left(\kappa^{\prime}\right), \quad T \circ \chi_{l}^{\mathrm{E}}(\kappa)=\chi_{l}\left(\kappa^{\prime}\right),
$$

where $\kappa^{\prime} \equiv \kappa\left(\Omega=e^{i \pi / 4}\right)$. Provided that, similar to what happened with the Wick rotation, one understands the transformation under $T$ of an integral over time as $T \circ \int d t f(t)=$ $\int d t T \circ f(t)$, it follows that $T$ maps the Euclidean to the Lorentzian action, but with different parameters, namely,

$$
T \circ I(\kappa)=S\left(\kappa^{\prime}\right) .
$$

It should be stressed that, in order to arrive at the above transformation rules for the constraints and the action, we have assumed that $T$ does not directly affect the coupling constants (i.e. $T \circ \kappa=\kappa$ ). Finally, one can readily check that the line element acquires a factor of $i$ under the action of $T$ :

$$
T \circ d s_{\mathrm{E}}^{2}=i d s^{2}
$$

that is, apart from mapping the Euclidean to the Lorentzian line element, $T$ induces a constant complex conformal transformation of the spacetime metric.

In view of these transformation laws, we conclude that $T=C^{\mathrm{F}} \circ R^{-1}$ provides in fact the Thiemann transform, since it is a map on the algebra of functions on phase space that preserves the Poisson bracket structure and sends the Euclidean constraints to their Lorentzian counterparts (although with complexified coupling constants $\kappa^{\prime}$ ).

It is worth remarking that the Thiemann transform attained in this way acts as the identity operator on all the Lagrange multipliers. If we had instead implemented the Wick rotation by means of the continuation in time $t \rightarrow-i t_{\mathrm{E}}$ and had denoted such a rotation as $\bar{R}$, to differentiate it from that obtained via a continuation of the lapse, then the transform $\bar{T}=C^{\mathrm{F}} \circ \bar{R}^{-1}$ would act on the Lagrange multipliers as

$$
\bar{T} \circ N_{\mathrm{E}}=-i N, \quad \bar{T} \circ u_{\mathrm{E}}^{l}=-i u^{l} .
$$

However, the action of $\bar{T}$ would actually coincide with that of the Thiemann transform $T$ on all the Euclidean phase space variables. As a consequence, both transforms could be considered equivalent modulo gauge, in the sense that their effects on any dynamical trajectory on the Euclidean constraint surface would only differ by a complex gauge transformation.

A different way of obtaining the Thiemann transform (7), which generalizes a proposal put forward by Ashtekar [2], is the following. We make the ansatz

$$
\mathcal{T}=\frac{\pi}{4} \sum_{\alpha} b_{\alpha} \int d^{3} x p_{\alpha}^{\mathrm{E}} q_{\mathrm{E}}^{\alpha}
$$

for the generator of the Thiemann transform $T$ and set $b_{\mathrm{g}}=2$ for the gravitational field, so that one recovers the definitions of $T$ in vacuum [1]. Furthermore, we also require that the remaining constants $b_{k}$ have the smallest absolute value such that $T$ maps the Euclidean constraints to the Lorentzian ones, up to a possible change in the coupling constants $\kappa$. By considering the transform of the scalar constraint, one then concludes that the 
unknown constants $b_{\alpha}$ must take the values $(-1)^{2 s_{\alpha}} s_{\alpha}$, so that they are determined by the spin of the fields $q_{\mathrm{E}}^{\alpha}$. A comparison of our ansatz for $\mathcal{T}$ with equation (6), shows that the transform derived in this way is precisely the Thiemann transform $T=C^{\mathrm{F}} \circ R^{-1}$ discussed above, as we wanted to show.

Ashtekar had already noted that the generator of the Thiemann transform had the form

$$
\frac{\pi}{4} \sum_{\alpha} s_{\alpha} \int d^{3} x p_{\alpha}^{\mathrm{E}} q_{\mathrm{E}}^{\alpha}
$$

and that this "might well be a reflection of a deeper structure underlying the generalized Wick transform" [2]. We have seen that the spin dependent factor is $(-1)^{2 s_{\alpha}} s_{\alpha}$ instead of $s_{\alpha}$, although a canonical transformation $(q, p) \rightarrow(-p, q)$ applied to the fermionic variables relates both expressions. The underlying structure that Ashtekar refers to is actually present. Indeed, $T$ preserves Poisson brackets and, therefore, it is sufficient to know the action of $T$ on the variables $q_{\mathrm{E}}^{\alpha}$ in order to determine $T$ completely. Moreover, since the Thiemann transform is a composition of an inverse Wick rotation $R^{-1}$ with the constant scale transformation of the fields $C^{\mathrm{F}}$, and the inverse Wick rotation acts on the variables $q_{\mathrm{E}}^{\alpha}$ as the trivial isomorphism $\mathcal{I}$ introduced in equation (更, we just need to know the transformation rules for the variables $q_{\mathrm{E}}^{\alpha}$ under $C^{\mathrm{F}}$. In other words, the Thiemann transform is completely determined by the action of constant scale transformations on the variables $q_{\mathrm{E}}^{\alpha}$, in which the dimensions of the fields $q_{\mathrm{E}}^{\alpha}$ play a central role. Finally, the covariance of the theory and the fact that the line element has dimension 2 relate the dimension and spin of the different fields. Summarizing, the requirement that the Thiemann transform preserves Poisson brackets, together with the covariance of the theory and the dimensional character of the spacetime line element, are the key structural features that underlie the Thiemann transform.

In [2], Ashtekar proposed an extension of the Thiemann transform, defined for vacuum general relativity, to gravity with a cosmological constant, coupled with a massive scalar field and an Abelian Yang-Mills field (this Abelian case corresponds to the limit of vanishing Yang-Mills constant $g$ ). Fermions were not included. Such an extension $T_{\mathrm{A}}$ was determined by the requirement that it maps the Euclidean action functional to the Lorentzian one with identical values of the coupling constants $\kappa$. In particular, this implies that $T_{\mathrm{A}}$ sends Euclidean to Lorentzian constraints via

$$
T_{\mathrm{A}} \circ \mathcal{S}_{\mathrm{E}}(\kappa)=\mathcal{S}(\kappa), \quad T_{\mathrm{A}} \circ \chi_{l}^{\mathrm{E}}(\kappa)=\chi_{l}(\kappa) .
$$

The transformation laws obtained by Ashtekar for phase space variables coincide with those found here for $T$ whereas, for coupling constants and Lagrange multipliers, they have the form

$$
\begin{gathered}
T_{\mathrm{A}} \circ \mu^{2}=-i \mu^{2}, \quad T_{\mathrm{A}} \circ \Lambda=-i \Lambda, \\
T_{\mathrm{A}} \circ N_{\mathrm{E}}=-N, \quad T_{\mathrm{A}} \circ N_{\mathrm{E}}^{a}=N^{a}, \quad T_{\mathrm{A}} \circ\left({ }^{4} A \cdot t\right)_{\mathrm{E}}=e^{i \pi / 4}\left({ }^{4} A \cdot t\right) .
\end{gathered}
$$

The difference in the sign of the transformation laws for the densitized lapse function under $T$ and $T_{\mathrm{A}}$ simply comes from a choice of a wrong relative sign between the Euclidean and Lorentzian scalar constraints as compared with the standard conventions. With the usual choice of sign, one has

$$
T_{\mathrm{A}} \circ N_{\mathrm{E}}=N
$$


On the other hand, the above transformation law for ${ }^{4} A \cdot t$ can actually be recovered in terms of the transform $T$ by noting that ${ }^{4} A \cdot t=g^{-1} \beta(g)$ (a relation that continues to be valid in the limit of vanishing $g$ ), taking into account that $T \circ \beta_{\mathrm{E}}(g)=\beta\left(g^{\prime}\right)$, and recalling that $T$ does not directly affect the coupling constants. Finally, notice that both $T$ and $T_{\mathrm{A}}$ leave the shift vector invariant. Therefore, it turns out that, for the matter content considered in [2], the only difference between $T$ and $T_{\mathrm{A}}$ is that the latter affects the coupling constants $\kappa$ while the former does not. In fact, it is easy to see that

$$
T_{\mathrm{A}}=C^{\kappa} \circ T
$$

where $C^{\kappa}$ is the constant scale transformation with factor $e^{i \pi / 4}$ restricted to act only on $\kappa$. Thus, while $T=C^{\mathrm{F}} \circ R^{-1}$ is a composition of an inverse Wick rotation and a constant scale transformation of the fields, $T_{\mathrm{A}}$ is equivalent to the composite of an inverse Wick rotation and a proper rescaling that affects all dimensional quantities, including the coupling constants, i.e.

$$
T_{\mathrm{A}}=C \circ R^{-1}
$$

with $C$ being the transformation $C_{\Omega}$, defined in equation (5), evaluated at $\Omega=e^{i \pi / 4}$.

\section{Conclusions and further comments}

The Thiemann transform was originally defined in vacuum general relativity as an automorphism on the algebra of functions on phase space that preserves the Poisson bracket structure and maps the constraints of Euclidean gravity to their Lorentzian counterparts. This transform was extended by Ashtekar to the case in which a scalar and a Maxwell field were present. In this work, we have further extended the Thiemann transform to the Ashtekar formulation for general relativity in the presence of general matter sources, namely, spin-1/2 fermions, a (non-Abelian) Yang-Mills field and a scalar field. We have proved that the action of the Thiemann transform on functions of the phase space variables is in fact equivalent to the result of an inverse Wick rotation and a constant scale transformation. Moreover, this equivalence also holds on functions that depend on the Lagrange multipliers as well (including the lapse function and the shift vector), provided that one performs the Wick rotation by means of an analytic continuation of the lapse and scales up all gravitational and matter fields, i.e. both the phase space variables and the Lagrange multipliers. For the spacetime metric, this complex rescaling amounts to a constant conformal transformation.

These results endow the Thiemann transform with a geometric interpretation. In addition, they provide, together with arguments based on general covariance and the dimensions of the line element, a simple explanation of the fact that the generator of the Thiemann transform is essentially determined by the spin of the different fields that are present in the theory [2].

Since the Thiemann transform $T$ relates the Euclidean and Lorentzian sectors of general relativity, one would expect that, were it possible to define a quantum analog of $T$, the Lorentzian quantum states could be obtained by transforming the solutions of the Euclidean quantum constraints [2]. In this way, the Thiemann transform could be employed in the quantum theory to circumvent the problem of imposing complicated reality 
conditions on the Ashtekar variables for gravity, because one can actually take these variables as real in the Euclidean sector (for an alternative procedure that avoids the use of a complex-valued Ashtekar connection in Lorentzian quantum gravity, see [16]). More precisely, if one uses the Pauli matrices $\tau_{A}^{i}{ }^{B}$ (with $i=1,2,3$ ) to express the Euclidean Ashtekar variables in the form [4]

$$
\sigma_{\mathrm{E}}^{a}=-\frac{i}{\sqrt{2}} \sigma_{i}^{a} \tau^{i}, \quad a_{a}^{\mathrm{E}}=-\frac{i}{2} a_{a}^{i} \tau^{i}
$$

it turns out that the densitized triad $\sigma_{i}^{a}$ and the connection $a_{a}^{i}$ can be chosen to be real. In the absence of fermions this is a consequence of the fact that the inclusion of matter sources does not modify the reality conditions for the gravitational variables, so that, as in vacuum Euclidean general relativity, one can restrict $\sigma_{i}^{a}$ and $a_{a}^{i}$ to be real [2]. In the presence of fermions, one can still work with real Euclidean triads $\sigma_{i}^{a}$. However, the reality conditions proposed in [4, 5] for the fermionic fields, together with relation (3) and the reality of the Euclidean extrinsic curvature, can be shown to imply that the connection $a_{a}^{i}$ has then an imaginary part equal to $-i \sigma_{a}^{i} \operatorname{tr}(y) / 4$, where $\sigma_{a}^{i}$ is the inverse of the densitized triad $\sigma_{i}^{a}$ and $y_{A B}$ is the function defined in equation (2). Nevertheless, one can easily recover a real connection by simply replacing $a_{a}^{i}$ with its real part, namely, with $a_{a}^{i}-\sigma_{a}^{i} \operatorname{tr}(y) / 4$. Indeed, it is not difficult to check that this replacement just amounts to a canonical transformation on the Euclidean phase space when it is accompanied with the following change of fermionic variables

$$
\left(\xi_{\mathrm{E}}, \rho_{\mathrm{E}}, \bar{\eta}_{\mathrm{E}}, \omega_{\mathrm{E}}\right) \rightarrow\left(\sigma^{1 / 2} \xi_{\mathrm{E}}, \sigma^{-1 / 2} \rho_{\mathrm{E}}, \sigma^{1 / 2} \bar{\eta}_{\mathrm{E}}, \sigma^{-1 / 2} \omega_{\mathrm{E}}\right)
$$

It is remarkable that the Thiemann transform may provide a way to extract the Lorentzian quantum physics from Euclidean general relativity. In this sense, we note that the Wick rotation for gravity is rather a formal technique for passing from the Lorentzian to the Euclidean path-integral approach. On the other hand, it is well known that quantum gravity is non-renormalizable, so that a change of scale cannot be simply absorbed by a redefinition of the coupling constants. However, we have seen that the combination of an inverse Wick rotation with a change of scale by a fixed factor of $e^{i \pi / 4}$ turns out to provide a rigorously defined transformation, which is just the Thiemann transform. Thus, if a quantum analogue of this transform exists, it should describe the behaviour of the quantum states under the simultaneous implementation of a Wick rotation and a fixed constant scale transformation of all fields, even if these two kinds of transformations are separately ill-defined in the quantum theory.

A line of research in which the Thiemann transform might find applications is the study of gravitational thermodynamics. Since statistical mechanics is naturally formulated in the Euclidean sector, the Thiemann transform might well be a useful tool to obtain the thermodynamical properties of Lorentzian gravity from an Euclidean formalism. One reason why classical solutions in gravity have intrinsic entropy is that the gravitational action is not scale invariant, but behaves instead like an area under a change of scale in the solutions [6]. As we have seen, this fact plays a key role in the existence of the Thiemann transform and its geometric interpretation. In addition, it is worth noticing that the thermodynamical properties of a gravitational system in vacuum are determined in the semiclassical approximation by the action of the solutions. This action comes 
entirely from a surface contribution that is given by the integral of the densitized trace of the extrinsic curvature [6]. Quite remarkably, this surface term has formally the same expression as the generator of the Thiemann transform in vacuum general relativity [1].

\section{Acknowledgments}

The authors are grateful to P. F. González Díaz for helpful conversations. This work was supported by funds provided by the DGICYT Project No. PB94-0107.

\section{Appendix}

Let us give the explicit expression of the self-dual action employed in [4, 5] to discuss the introduction of matter sources in the Ashtekar formalism for gravity. In the Hamiltonian formulation, this action can be written in the form

$$
\begin{aligned}
S^{\epsilon}=\int d t d^{3} x & \left\{\epsilon^{-1} \sqrt{2} \operatorname{tr}\left(\sigma^{a} \dot{a}_{a}\right)+\pi_{\phi} \dot{\phi}+\operatorname{tr}\left(E^{a} \dot{A}_{a}\right)+\rho_{A} \dot{\xi}^{A}+\omega_{A} \dot{\bar{\eta}}^{A}\right. \\
& \left.+g \operatorname{tr}\left[\left({ }^{4} A \cdot t\right) G^{\epsilon}\right]+\operatorname{tr}\left[\left({ }^{4} a \cdot t\right) \mathcal{G}^{\epsilon}\right]+N^{a} \mathcal{V}_{a}^{\epsilon}+N \mathcal{S}^{\epsilon}\right\},
\end{aligned}
$$

where traces have been displayed explicitly. In this formula, $\epsilon=-i$ for the Lorentzian theory, while the Euclidean action is obtained by setting $\epsilon=1$. The Gauss constraint associated with the Yang-Mills connection takes the same expression in the Lorentzian and Euclidean sectors, namely,

$$
G^{\epsilon}=g^{-1} D_{a} E^{a} .
$$

The Gauss constraint associated with the Ashtekar connection and the vector constraint can be written as

$$
\begin{aligned}
\mathcal{G}_{A B}^{\epsilon} & =\frac{1}{\epsilon} \sqrt{2} \mathcal{D}_{a} \sigma_{A B}^{a}-\rho_{(A} \xi_{B)}-\omega_{(A} \bar{\eta}_{B)}, \\
\mathcal{V}_{a}^{\epsilon} & =-\frac{1}{\epsilon} \sqrt{2} \operatorname{tr}\left(\sigma^{b} F_{a b}\right)-\rho_{A} \mathcal{D}_{a} \xi^{A}-\omega_{A} \mathcal{D}_{a} \bar{\eta}^{A}-\pi_{\phi} \partial_{a} \phi-\frac{1}{2} \operatorname{tr}\left(E^{b} B_{a b}\right) .
\end{aligned}
$$

Finally, the scalar constraint is given by

$$
\begin{aligned}
\epsilon^{2} \mathcal{S}^{\epsilon}= & \operatorname{tr}\left(\sigma^{a} \sigma^{b} F_{a b}\right)+\frac{1}{8 \sigma^{2}} \operatorname{tr}\left(\sigma^{a} \sigma^{c}\right) \operatorname{tr}\left(\sigma^{b} \sigma^{d}\right) \operatorname{tr}\left(\epsilon^{2} E_{a b} E_{c d}-B_{a b} B_{c d}\right) \\
& -i m\left(\sigma^{2} \xi^{A} \bar{\eta}_{A}+\epsilon^{2} \rho^{A} \omega_{A}\right)+\epsilon \sqrt{2} \sigma_{A}^{a}{ }_{A}^{B}\left(\rho_{B} \mathcal{D}_{a} \xi^{A}+\omega_{B} \mathcal{D}_{a} \bar{\eta}^{A}\right) \\
& +\epsilon^{2} \frac{\pi_{\phi}^{2}}{16 \pi}-4 \pi \sigma^{2} \mu^{2} \phi^{2}+4 \pi \operatorname{tr}\left(\sigma^{a} \sigma^{b}\right) \partial_{a} \phi \partial_{b} \phi-\sigma^{2} \Lambda .
\end{aligned}
$$

In these expressions, $D_{a}$ is the derivative operator associated with the Yang-Mills connection $A_{a}$, and $B_{a b}$ is twice its curvature:

$$
\begin{aligned}
D_{a} E^{a} & =\partial_{a} E^{a}+g\left[A_{a}, E^{a}\right], \\
B_{a b} & =2\left(\partial_{a} A_{b}-\partial_{b} A_{a}+g\left[A_{a}, A_{b}\right]\right) .
\end{aligned}
$$


In addition, $\mathcal{D}_{a}$ is the derivative operator associated with the Ashtekar connection $a_{a}$, and $F_{a b}$ is its curvature, i.e.

$$
\begin{aligned}
\mathcal{D}_{a} \xi^{A} & =\partial_{a} \xi^{A}-a_{a B}^{A} \xi^{B}, \\
F_{a b A}{ }^{B} & =\partial_{a} a_{b A}{ }^{B}-\partial_{b} a_{a A}{ }^{B}+a_{a A}{ }^{C} a_{b C}{ }^{B}-a_{b A}{ }^{C} a_{a C}{ }^{B} .
\end{aligned}
$$

Finally, $\sigma=\sqrt{\operatorname{det}\left(\sigma^{a}\right)}$ and

$$
E_{a b}=\eta_{a b c} E^{c},
$$

where $\eta_{a b c}$ is the c-number Levi-Civita form-density.

\section{References}

[1] Thiemann T 1996 Class. Quantum Grav. 131383

[2] Ashtekar A 1996 Phys. Rev. D 532865

[3] Mena Marugán G A 1997 Geometric interpretation of Thiemann's generalized Wick transform Preprint gr-qc/9705031, Grav. Comol. to appear

[4] Ashtekar A 1991 Lectures on non-perturbative canonical gravity ed L Z Fang and R Ruffini (Singapore: World Scientific)

[5] Ashtekar A, Romano J D and Tate R S 1989 Phys. Rev. D 402572

[6] Hawking S W 1979 The path-integral approach to quantum gravity General Relativity.An Einstein Centenary Survey ed S W Hawking and W Israel (Cambridge: Cambridge University Press)

[7] Gibbons G W, Hawking S W and Perry M J 1978 Nucl. Phys. B 138141

[8] Mazur P O and Mottola E 1990 Nucl. Phys. B 341187

[9] Greensite J 1993 Phys. Lett. B 30034

Carlini A and Greensite J 1994 Phys. Rev. D 49866

Ivashchuk V D 1997 Grav. Cosmol. 38

[10] See, for instance, Halliwell J J and Hawking S W 1985 Phys. Rev. D 311777

[11] See, for instance, D'Eath P D and Halliwell J J 1987 Phys. Rev. D 351100

[12] Jacobson T 1988 Class. Quantum Grav. 5 L143

[13] Faddeev L D and Slavnov A A 1980 Gauge Fields: Introduction to Quantum Theory (New York: Benjamin)

[14] Hawking S W 1984 Nucl. Phys. B 239257

[15] Mena Marugán G A 1997 Proc. Fifth Wigner Symp. (Singapore: World Scientific) to be published

[16] Thiemann T 1998 Class. Quantum Grav. 15 839; 1998 Class. Quantum Grav. 15 875; 1998 Class. Quantum Grav. 151281 